\begin{tabular}{|c|c|c|c|c|c|c|}
\hline Variable & Pre-bundle period & p-value & Post-bundle period & Bundle not used & $\mathrm{p}$-value & Bundle used \\
\hline \multicolumn{7}{|l|}{ DEMOGRAPHIC VARIABLES } \\
\hline Number of patients & $459(51.4 \%)$ & $\mathrm{n} / \mathrm{a}$ & $434(48.6 \%)$ & $205(53.4 \%)$ & $\mathrm{n} / \mathrm{a}$ & $179(46.6 \%)$ \\
\hline Age (years) & $64.7[63.0-66.4]$ & 0.772 & $64.4[62.7-66.0]$ & $65.8[63.4-68.1]$ & 0.043 & $62.2[59.5-64.8]$ \\
\hline Male & $264(57.8 \%)$ & 0.530 & $266(61.4 \%)$ & $123(60.0 \%)$ & 0.395 & $113(63.1 \%)$ \\
\hline Female & $193(42.2 \%)$ & & $167(38.6 \%)$ & $82(40.0 \%)$ & & $66(36.9 \%)$ \\
\hline GBS score & $6.3[0-19]$ & 0.011 & $7.4[0-20]$ & 7 [0-18] & 0.141 & $8.0[0-20]$ \\
\hline Varices & $27(7.3 \%)$ & 0.007 & $51(13.3 \%)$ & $24(13 \%)$ & 0.733 & $24(14 \%)$ \\
\hline \multicolumn{7}{|l|}{ BUNDLE VARIABLES } \\
\hline Blatchford (GBS) recorded & $163(38.4 \%)$ & $<0.001$ & $225(57.3 \%)$ & $86(42.4 \%)$ & $<0.001$ & $122(74.0 \%)$ \\
\hline Target haemoglobin stated & $53(35.3 \%)$ & 0.083 & $78(44.8 \%)$ & $34(36.6 \%)$ & 0.039 & $35(53.0 \%)$ \\
\hline Transfusion threshold appropriate & $58(13.3 \%)$ & 0.012 & $83(19.6 \%)$ & $40(19.6 \%)$ & 0.880 & $36(20.2 \%)$ \\
\hline Rebleeding plan & $121(33.2 \%)$ & $<0.001$ & $196(50.7 \%)$ & $83(44.9 \%)$ & 0.028 & 95 (56.55\%) \\
\hline
\end{tabular}

Conclusions Implementation of the UK AUGIB bundle in Scottish hospitals resulted in significant improvements in quality standards including documentation of risk scoring, target haemoglobin, transfusion thresholds and re-bleed plan.

\section{PTH-013 DEVELOPMENT OF THE UPPER GI RECORDED IMAGE QUALITY INDEX (UGI-RIQI) SCORE AND QUALITY ASSURANCE TOOL}

${ }^{1}$ Thomas Dove*, ${ }^{2}$ Esther Hawkes, ${ }^{1}$ James Berrill, ${ }^{1}$ Bee Lee, ${ }^{1}$ Peter Neville, ${ }^{1}$ Neil Hawkes. ${ }^{1}$ Cwm Taf University Health Board, Cardiff, UK; ${ }^{2}$ University of Southampton, Southampton, $U K$

\subsection{6/gutjnl-2019-BSGAbstracts.38}

Background Endoscopic images saved on the Electronic Reporting System are the only visible representation of completeness of examination and pathological findings. Together with the endoscopy report these become the only reference for other clinicians not present at the original endoscopy on which to base further decisions. Standards for image recording form a component part of the Quality Standards in Upper Gastrointestinal (UGI) endoscopy. ${ }^{1}$

Aims and methods We aimed to develop a systematic scoring system for quality of images recorded at UGI endoscopy and validate this UGI Recorded Image Quality Index (UGI-RIQI) scoring system. We searched the HICSS Endoscopic Reporting System for endoscopists performing regular UGI endoscopy $(n=14)$ between January and June 2018. All images and the endoscopy report for the first 10 cases with pathological findings for each endoscopist were obtained, ordered into folders and the data anonymised. An UGI-RIQI scoresheet was devised, based on the validated lower GI RIQI tool, ${ }^{2}{ }^{3}$ assessing 4 domains: Representation, Image Labelling, Extent of examination and Image Quality, and the clinical utility (CU) of the image set - rating its ability to inform further decisionmaking. The UGI-RIQI total score range was 0 to 12.140 image sets were scored by 3 independent assessors. Cohen's kappa values for intra observer variation were calculated for individual domains and total RIQI scores. These results informed recommended RIQI standard levels of performance. The correlation of these levels and CU scores was tested with Spearman's test.

Results 140 data sets were reviewed by 3 assessors generating 420 domain scores. Inter-rater agreement (IRA) for assessors for the total RIQI score were in the moderate to good range
$(0.6,0.46$ and 0.47$)$. Performance levels were defined in terms of total RIQI score: poor 0-6, below standard 7-8 and meets standards 9-12. The correlation between the derived RIQI levels and clinical utility scores were high $(0.71,0.64$ and 0.71$)$. Conclusions The UGI-RIQI tool provides a method for assessing the quality of image capture across ten procedures with scores in 4 domains. The UGI-RIQI score correlates well with clinical utility of the images, with acceptable inter-rater reliability. It shows potential both as an audit and training tool to improve performance in this area of endoscopic practice.

\section{REFERENCES}

1. Beg S, et al. Gut 2017;0:1-14. doi:10.1136/gutjnl-2017-314109.

2. Samuel D, et al. Gut 2016; 65:Suppl 1 A224-A225 doi:10.1136/gutjnl-2016312388.420 .

3. Samuel D, et al. Gut 2016;65:Suppl 1 A278 doi:10.1136/gutjnl-2016312388.522 .

\section{PTH-014 DIAGNOSTIC PERFORMANCE OF ERCP GUIDED BILIARY BRUSH CYTOLOGY- EXPERIENCE FROM A NON-HPB CENTRE IN UK}

Mohamed Elzubier*, Debasis Majumdar, Vikramjit Mitra. South Tees Hospital, Middlesbrough, UK

\subsection{6/gutjnl-2019-BSGAbstracts.39}

Introduction Biliary brush cytology is an important investigation in the assessment of bile duct strictures. A meta-analysis ${ }^{1}$ showed that the pooled sensitivities and specificities of biliary brush cytology for the diagnosis of malignant biliary strictures were $45 \%$ and $99 \%$ respectively when done in tertiary centres. The aim of this study was to evaluate the diagnostic performance of ERCP guided brush cytology for the assessment of biliary strictures in our organisation.

Methods We carried out a retrospective review of all biliary brushings (identified from our endoscopy database) obtained during ERCP between January 2012 and April 2017. Data collected included patient demographics, cross-sectional imaging, cytopathological classification (based on locally agreed terminology) and treatment modality. Final diagnosis was confirmed from biliary brush cytology, histology obtained by other methods (endoscopic ultrasound, cholangioscopy, PTC or ultrasound guided biopsy), surgical resection specimens or cross-sectional imaging discussed at MDT setting (if histology negative). Patients were followed up for at least 6 months. 
Results 215 patients (median age 73 years, 50.7\% males) underwent 250 procedures - 153 patients had a final diagnosis of malignancy and underwent 163 procedures, 62 patients had a final diagnosis of benign pathology and underwent 87 procedures. 246 (98.4\%) samples were deemed adequate for cytological analysis.

Results of biliary brushings are enclosed in the table 1

\begin{tabular}{|c|c|c|c|c|c|}
\hline & Inadequate & Benign & Atypical & Suspicious & Malignant \\
\hline $\begin{array}{l}\text { Final } \\
\text { diagnosis of } \\
\text { malignancy } \\
n=163\end{array}$ & 4 & 38 & 33 & 28 & 60 \\
\hline $\begin{array}{l}\text { Final } \\
\text { diagnosis } \\
\text { benign } n=87\end{array}$ & 0 & 82 & 1 & & 0 \\
\hline \multicolumn{4}{|l|}{ Key } & & \\
\hline & & \multicolumn{2}{|c|}{ False negatives } & & \\
\hline & & \multicolumn{2}{|c|}{ True positives } & & \\
\hline & & \multicolumn{2}{|c|}{ True negatives } & & \\
\hline & & \multicolumn{2}{|c|}{ False positives } & & \\
\hline
\end{tabular}

\section{Abstract PTH-014 Table 1}

Sensitivity, specificity, positive predictive value (PPV) and negative predictive value (NPV) were 55.1\%, 95.4\%, 95.6\%, and $53.9 \%$ respectively [with atypia included as false negative in patients with cancer as final diagnosis]. The overall accuracy of the test was $69.3 \%$.

Conclusion Our study confirms that ERCP guided brush cytology for assessment of biliary stricture can be effective in a non-HPB centre with comparable rates of diagnostic yield to tertiary HPB centres. The low sensitivity (55.1\%) indicates that biliary brushing alone is insufficient to rule out malignancy in a significant proportion of patients with a final diagnosis of malignancy. Further adjuncts like EUS and cholangioscopy are required to increase the diagnostic yield in biliary strictures.

\section{REFERENCES}

1. Navaneethan U, Njei B, Lourdusamy $V$, et al. Comparative effectiveness of biliary brush cytology and intraductal biopsy for detection of malignant biliary strictures: a systematic review and meta-analysis. Gastrointest Endosc 2015;81:168-76.

\section{PTH-015 INFLAMMATORY REACTION PATTERNS AND MOLECULAR GENETICS IN HIGH-GRADE COLORECTAL ADENOMAS}

Andrew Emmanuel*, Salvador Diaz-Cano, Shraddha Gulati, Savvas Papagrigoriadis, Bu Hayee, Amyn Haji. King's College Hospital NHS Foundtaion Trust, London, UK

\subsection{6/gutjnl-2019-BSGAbstracts.40}

Introduction Inflammatory stromal changes are frequent findings in large colorectal adenomas that can contribute to neoplastic progression, but their role is not fully established in high-grade adenomas of the colon as predictors of coexistent invasive malignancy.

Methods ER of large colorectal adenomas (2011-2016) were analysed. A subset containing high-grade dysplasia, intramucosal cancer or invasive cancer was identified and subjected to detailed histopathological analysis: ulceration, distribution of high-grade dysplasia, dysplastic nuclear grade, presence/distribution of necrosis, and distribution of tumour-infiltrating lymphocytes (TIL). Microdissection, DNA extraction and nextgeneration sequencing using a human clinically relevant tumour panel of 24 genes were performed separately for two areas with the highest morphological grade from each lesion.

Results ER was performed for 418 large $(\geq 20 \mathrm{~mm})$ adenomas. Histopathological genetic evaluation was available in 70 high grade cases. The extension of TIL positively correlated with the presence of coexistent adenocarcinoma, along with multifocal intraluminal necrosis and high nuclear grade. The presence of multifocal high-grade dysplasia was driven by different cooperative sets of genetic abnormalities regarding the TIL pattern: high-impact (TP53), and moderate impact (FLT4, ERBB2, RET/RAS/RAF/ERK) for an interface pattern, and high-impact (TP53, PDFGRA, FGFR3), and moderate impact (TP53, ERBB2, KRAS, RET/FGFR3) for an interstitial pattern. Conclusions A more extensive lymphocytic response contributes to the progression of adenomas and is associated with morphological changes of increased risk of adenocarcinoma (multifocal necrosis and high nuclear grade and abnormal TP53). An interface pattern expresses proliferative (receptor kinase) and vascular (FLT4) profile and the interstitial pattern outlines predominantly stromal (PDGFRA, FGFR3) features.

\section{PTH-016 RECURRENCE AFTER ADVANCED COLORECTAL ENDOSCOPIC RESECTION RESULTS IN A SUBSTANTIAL COST BURDEN}

Andrew Emmanuel*, Sophie Williams, Shraddha Gulati, Christo Lapa, Nishmi Gunasingam, Margaret Burt, Bu Hayee, Amyn Haji. King's College Hospital NHS Foundtaion Trust, London, UK

\subsection{6/gutjnl-2019-BSGAbstracts.41}

Introduction Little is known about the cost of treating recurrence after colorectal endoscopic resection (ER). Endoscopic mucosal resection (EMR) has proven efficacy and safety and is the procedure of choice for the majority of western endoscopists. However, recurrence is not infrequent but no studies examine the cost associate with ER taking into account treatment for recurrence. We evaluated the total cost of ER at a tertiary centre.

Methods ER (EMR, ESD and Hybrid ESD) of large $(\geq 20$ $\mathrm{mm}$ ) colorectal tumours with at least the first surveillance colonoscopy were included. Surveillance was performed at 3-6 months and 12 months. Procedure costs were based on NHS national tariffs. Total costs included the cost of all endoscopic or surgical treatments and surveillance to 12 months. Cost of treatment for patients with recurrent adenomas and those without were compared.

Results Of 626 ER of large colorectal tumours, 473 (98\% of eligible patients) had undergone at least the first surveillance colonoscopy. Overall, recurrence occurred in 69 (14.7\%). Patients with recurrence required a median of 2 ERs (range 2-6). 8 patients required surgery for recurrence. Mean cost of treating those with recurrence was $£ 3976$ versus $£ 1539$ for those without $(\mathrm{p}<0.001) .51 \%$ of lesions had been subjected to prior failed attempts at resection or extensive $(\geq 6)$ biopsy sampling and these were significantly more likely to recur (OR 3.8, 95\% CI 2.1-6.9, p<0.001). The cost of treating these patients was significantly more than those with minimal or no manipulation $(\mathrm{p}=0.001)$. Recurrence was less likely after ESD (OR 0.25, 95\% CI 0.08-0.82, $\mathrm{p}=0.01$ ). 